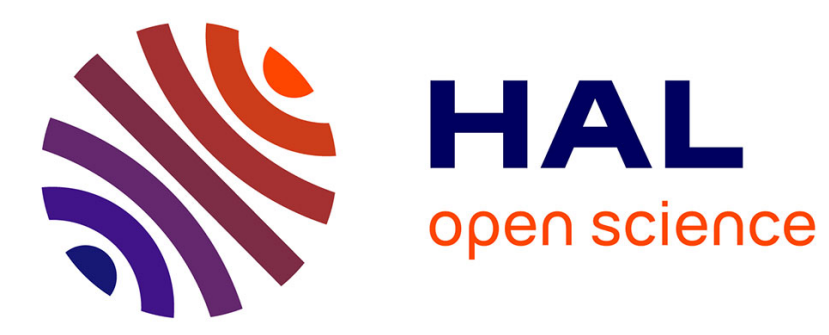

\title{
Relating circular dichroism to atomic structure by means of MD simulations and computed CD spectra with $\alpha$-peptoids as an example
}

Nicholus Bhattacharjee, Lionel Perrin, Franck Jolibois

\section{- To cite this version:}

Nicholus Bhattacharjee, Lionel Perrin, Franck Jolibois. Relating circular dichroism to atomic structure by means of MD simulations and computed CD spectra with $\alpha$-peptoids as an example. Physical Chemistry Chemical Physics, 2020, 22 (23), pp.13192-13200. 10.1039/D0CP01336F . hal-03006401

\section{HAL Id: hal-03006401 https://hal.science/hal-03006401}

Submitted on 16 Nov 2020

HAL is a multi-disciplinary open access archive for the deposit and dissemination of scientific research documents, whether they are published or not. The documents may come from teaching and research institutions in France or abroad, or from public or private research centers.
L'archive ouverte pluridisciplinaire HAL, est destinée au dépôt et à la diffusion de documents scientifiques de niveau recherche, publiés ou non, émanant des établissements d'enseignement et de recherche français ou étrangers, des laboratoires publics ou privés. 


\section{ARTICLE}

\section{Relating circular dichroism to atomic structure by means of MD simulations and computed CD spectra: the example $\alpha$-peptoids}

Received 00th January 20xx, Accepted 00th January 20xx DOI: $10.1039 / x 0 \times x 00000 x$

\begin{abstract}
Nicholus Bhattacharjee, ${ }^{a, b}$ Lionel Perrin $^{b}$ and Franck Jolibois*a
Classical molecular dynamics simulations have been combined to quantum calculations of CD spectra in order to fruitfully relate the experimental CD spectra, not only to the overall conformation of chiral $\alpha$-peptoids, but also to its structure at the atomic scale, including the dihedral feature of the backbone $(\psi, \varphi)$ and the orientation of the chiral side-chain $(\chi 1)$. These simulations have been performed up to the hexamer $\mathrm{Ac}$-(stbe) ${ }_{6}-\mathrm{CO}_{2} \mathrm{tBu}$. We have shown that number of states has a significant impact on the shape of the spectrum below $215 \mathrm{~nm}$. The number of states computed is also critical to simulate the spectra of long oligomer. While 10 to 20 states are sufficient to simulate the CD spectra of short oligomers, 100 states or more are mandatory to converge the CD spectrum shape for longer oligomers. The conformational sampling and the analysis of the intramolecular interactions responsible for the specific folding of the objects have been jointly explored by means of Replica Exchange MD and DFT calculations.
\end{abstract}

\section{Introduction}

Peptoids are polyglycines like peptides with $\mathrm{N}$-substituted amide with specific chemical groups. While they are very close mimics of peptides, peptoids have lost both their $\mathrm{N}-\mathrm{H} \mathrm{H}$-bond donors and asymmetric $\alpha$ carbons: two characteristics that specifically control peptide folding. In peptoids, as the $\alpha$ carbon is achiral, the stereogenic information can be re-introduced via the chemical group attached to the amide nitrogen atom. ${ }^{1}$ Because of the loss of the $\mathrm{H}$-bonding network and of the intrinsic flexibility of long oligomers, the experimental determination of three-dimensional (3D) structures is significantly hampered. Most of the time, experimentally available 3D structures are based on X-ray diffraction of medium sized crystalized molecules. ${ }^{2}$ Although, 1D and 2D NMR spectroscopy is routinely employed to characterize the primary sequence of such oligomers, their 3D structures could only be determined in few cases. Indeed, the loss of the amide proton makes multidimensional NMR technics employed to resolve 3D structure useless. Interestingly, when chiral properties are reintroduce by a chiral side chain appended to the nitrogen atom of the backbone, Electronic Circular Dichroism (ECD) allows to determine the overall secondary structures of the foldamers ${ }^{1}$ though this analytical technic has some intrinsic limitations. ${ }^{3}$

\footnotetext{
a. Université de Toulouse-INSA-UPS, LPCNO, CNRS UMR 5215, 135 av. Rangueil, F31077, Toulouse, France.

b. Université de Lyon, Université Claude Bernard Lyon 1, CPE Lyon, INSA Lyon, ICBMS, CNRS UMR 5246, Equipe ITEMM, Bât. Lederer, 43 Bd. du 11 Novembre 1918, 69622 Villeurbanne, France.

Electronic Supplementary Information (ESI) available: [Tables of geometry trends, Figures of probability distributions, of identification and occurrence of weak interactions, Cartesian coordinates of most relevant oligopeptoids]. See DOI: $10.1039 / x 0 x \times 00000 x$
}

Classical Molecular Dynamics (MD) simulations combined to the calculations of spectroscopic data using quantum chemical methods can be employed nowadays to obtain further insight into the 3D structures at the atomic scale of these oligomers. On the one hand, analyses of MD trajectories allow describing some specific structural characteristics. On the other hand, spectroscopic response computed at the quantum level can be used to precisely reproduce experimental spectroscopic data and to highlight unambiguous specific conformations or intramolecular interactions responsible of the spectral signatures (eg ECD (CD) or NMR). Electronic circular dichroism is a property of chiral compounds to absorb left and right circularly polarized light. CD spectroscopy has been intensively used in chemical, biochemical and biophysical analysis of chiral compounds in order to extract overall three-dimensional information. Because of the complexity of phenomena hidden behind $C D$ spectrum signatures, only little information can be extracted at the atomic scale from experimental $C D$ spectrum solely.

Recently, a new type of side chain carrying only aliphatic chemical groups has been proposed by experimental chemists ${ }^{4}$ in order to introduce chirality to peptoid-based foldamers. Among the side chains proposed, the new tert-butylethyl (stbe) is not aromatic. This is of primary importance because the CD spectral signature arising from such oligomer is only due to electronic excitations located in the peptoid backbone and arises from the peptide linkage. For residues bearing aromatic functions (naphthylethyl, 5,6 phenylethyl, 7,8 (benzyltriazolium) ethyl, 9,10 ...), CD spectra are more complicated because electronic excitations imply both peptoid backbone and side chains aromatic moieties. 


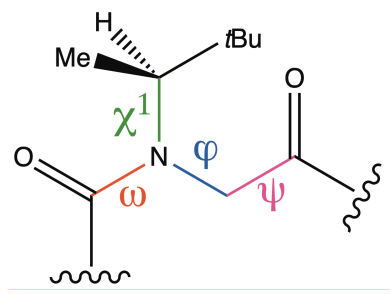

Scheme 1

This not only intricates the interpretation of spectral characteristics but also increases computational efforts of the quantum chemical simulations of the $C D$ spectrum of such oligomers. We propose to establish a general methodology that combines both MD simulations and computed spectroscopic properties in order to interpret the structure at the atomic scale of the different $C D$ spectra of oligopeptoids supporting the saturated chiral tert-butylethyl side chain (Scheme 1). These systems are used both as an object of concern and a prototypical chiral foldamer. By simulating as precisely as possible the spectra of chiral $\alpha$-peptoid foldamers of various lengths and by direct comparison with experimental data, we intend to reveal a set of specific conformational properties that explain the spectral characteristics. In a second step, molecular dynamics simulation of a specific type of oligomer has also been employed in order to complete the conformational properties that characterize this family of chiral peptoids. Due to the computational cost of quantum calculations, molecular dynamic simulations have also been employed to compare the fluxional character of chiral vs achiral residues.

\section{Modelling strategy and computational details}

All quantum chemical calculations were performed using Gaussian 09 suite of programs. ${ }^{11}$ Geometry optimization of studied foldamers have been achieved using the hybrid Density Functional Theory approach B3LYP ${ }^{12,13}$ with Pople type double $\zeta$ basis sets augmented by polarization and diffuse functions on heavy atoms and polarization functions only on hydrogen atoms (namely $6-31+\mathrm{G}(d, p)) \cdot{ }^{14,15}$ In order to validate this computational approach, M06-2X $\mathrm{X}^{16}$ functional has also been tested with Pople type triple $\zeta$ basis sets augmented by polarization functions on carbon and hydrogen (namely 6$311 \mathrm{G}(d, p)$ ) plus diffuse functions on nitrogen and oxygen (namely 6-311+G(d)).

In order to calculate electronic excitation wavelengths ( $\lambda_{\text {TD-DFT }}$ ) and circular dichroism (CD) parameter ( $\left.R^{v e l}\right)$, TD-B3LYP calculations have been performed using $6-31+G(d, p)$ basis sets. For geometry optimizations and electronic excitation computations, the integral equation formalism model (IEFPCM) has been employed in order to simulate bulk solvent effects (i.e. acetonitrile; $\varepsilon=35.688$ and $\varepsilon$ (inf) $=1.806874$ ). Finally, in order to compare theoretical and experimental data, CD spectra have been simulated by performing convolution with Gaussian type functions of each calculated transition and summing these Gaussian functions on the whole wavelength range (Eq. 1 and Figure 1).

$$
I\left(\lambda_{\text {Spec }}\right)=\sum_{i=1}^{\text {NStates }} R_{i}^{\text {vel }} e^{-\frac{\left(\lambda_{\text {Spec }}-\lambda_{T D-D F T}^{i}\right)^{2}}{B W}}
$$

In Eq. $1 I\left(\lambda_{\text {Spec }}\right)$ is the intensity of the simulated spectrum at $\lambda_{\text {Spec }} . \lambda_{T D-D F T}^{i}$ and $R_{i}^{\text {vel }}$ are the wavelength and the rotatory strength velocity of the $i^{\text {th }}$ TD-DFT excited state, respectively. The parameter BW was arbitrary set to $100 \mathrm{~nm}^{2}$ in order to achieve a fixed width at half-length close to $17 \mathrm{~nm}$ that also corresponds to a broadening ca $0.5 \mathrm{eV}$. No correction (red or blue shift) has been applied on the theoretical wavelengths when simulating whole CD spectra.

For Molecular Dynamics (MD) simulations, the Generalized AMBER Force Field (GAFF) ${ }^{17}$ parameters were used to describe the potential of tert-butyl (tBu) and tert-butylethyl (stbe) peptoid monomers. The RESP charges were generated using RED server ${ }^{18}$ followed by the antechamber module ${ }^{19}$ of AMBER to make the parameter file. The peptoid polymers were capped by acetyl group at N-terminus and N,N-dimethyl at C-terminus. Initially the $\omega$ dihedral angle describing the peptide bond was kept at $0^{\circ}$ in agreement with a cis peptide conformation. To assess the chirality induction, the $\varphi$ dihedral angles for stbe monomers were kept at negative values while such values for tBu were altered to generate four different conformations. The peptoid polymer was solvated in acetonitrile box with a buffer of $20 \AA$ towards each direction.

All Molecular Dynamics simulations were performed using NAMD software package. ${ }^{20}$ A time step of $1 \mathrm{fs}$ was applied for each simulation. A cutoff of $14 \AA$ was applied for non-bonded interactions. Particle Mesh Ewald summation was applied for electrostatic interactions. The system was initially minimized in the NVT ensemble. During this NVT thermalization the temperature was gradually raised from $5 \mathrm{~K}$ to $315 \mathrm{~K}$ with an increment of $10 \mathrm{~K}$. At each temperature 5000 steps (5 ps) of simulation was performed. This was followed by 200 ps of NVT equilibration at $300 \mathrm{~K}$. Thereafter the system was subjected to 400 ps of equilibration in the NPT ensemble. The temperature was kept fixed at $300 \mathrm{~K}$ through Langevin dynamics with a damping coefficient of $5 / p s$ while the pressure was controlled at $1 \mathrm{~atm}$ using Nose-Hoover Langevin piston.

From the equilibrated system two initial production runs of each $10 \mathrm{~ns}$ were performed. The resultant snapshots were subjected to another two similar production runs each. A final production run of 50 ns was performed from the final snapshot of the initial production run. The trajectory was stored for every 2 ps. The trajectory from this final production run was used for calculation of all equilibrium properties. A similar protocol was followed for oligopeptide decamer starting from four different conformations.

The Replica Exchange Molecular Dynamics (REMD) simulations ${ }^{21-23}$ were performed for a temperature range of 300 to $600 \mathrm{~K}$. The generation of temperature series was done using scheme developed by Patrikson et.al. ${ }^{24}$ This allows a uniform exchange probability for all replicas during REMD simulation. The targeted exchange probability, $P_{\text {exch, }}$ was set at a value of 0.3 . For all four decamers showing different conformations, the final production snapshots were used as input structure. 50 
numbers of replicas were used with 50 nanoseconds of trajectory for each replica.

\section{Results and discussion}

$C D$ computations on monomer - Solvent effect and cis vs trans effect. Our first attempt to calculate electronic circular dichroism has been focused on the Ac-stbe- $\mathrm{CO}_{2} \mathrm{tBu}$ monomer compound. For this molecule, the influence of the conformation and of the solvent on the $C D$ spectrum features have been analyzed. According to experimental results, ${ }^{4} \mathrm{Ac}$-stbe- $\mathrm{CO}_{2} \mathrm{tBu}$ exhibits an equilibrium constant $K_{\text {cis/trans }}$ value ranging between 0.64 and 0.77 depending on the solvent. These $K_{\text {cis/trans }}$ values indicate that the solution is roughly composed by $40 \%$ and $60 \%$ of cis and trans peptide conformation, respectively. The difference in Gibbs energy between these two conformations is thus small, less than $0.5 \mathrm{kcal} \mathrm{mol}^{-1}$. Geometry optimizations of both cis and trans isomers have been performed using both B3LYP and M06-2X density functionals (see Computational Details). In addition to the peptide bond conformation, the influence of $\chi 1(C(i-1)-N-N C \alpha-N C \beta)$ dihedral angle has been considered by defining three initial confirmations having $\chi 1$ torsions around $10^{\circ}, 110^{\circ}$ and $-110^{\circ}$. All attempts to optimise geometry starting from $\chi 1=10^{\circ}$ have led to structure with a $\chi 1$ values ranging from 70 to $100^{\circ}$.


Fig. 1 Simulation of $C D$ spectra of $A c-(\text { stbe })_{6}-\operatorname{CoOtBu}$. Without $(A)$ and with $(B)$ convolution with Gaussian functions on each excitation peak accordingly to Eq. 1.
Among the two more stable isomers, the trans isomer is $0.2 \mathrm{kcal}$ $\mathrm{mol}^{-1}$ (B3LYP) and $0.3 \mathrm{kcal} \mathrm{mol}^{-1}$ (M06-2X) more stable than the cis one. This trend is in excellent agreement with the $K_{\text {cis/trans }}$ experimental values. Moreover, the preference for $\chi 1$ angle value around $-110^{\circ}$ is consistent with the $X$-ray data available for the pentamer (Ac-(stbe) $\left.)_{5}-\operatorname{COOtBu}\right)^{4}$ that shows $\chi 1$ torsion angle values ranging from $-100^{\circ}$ to $-105^{\circ}$. When solvent effects are implicitly taken into account using either the $\mathrm{SMD}^{25}$ or IEFPCM ${ }^{26}$ model, the relative stability of the trans isomer over the cis is maintained in acetonitrile, methanol or chloroform (data not shown). This set of results validates either B3LYP or M06-2X as a proper density functional to study oligopeptoids. As the main objective is to establish a computational approach that enable to simulate $C D$ spectra of foldamers of various length, two computational approaches including or not solvent effects have been compared. Isomers characterized by $\chi 1=-$ $110^{\circ}$ have only been considered based on stability consideration discussed above.

Figure 2 gives the average spectra calculated by summing $40 \%$ of the cis spectrum with $60 \%$ of the trans one. In order to compare both DFT methods, all intensities have been scaled in order to obtain a maximum intensity of 1 . Qualitatively, both DFT approaches lead to the same CD spectra. For the gas phase, both methods exhibit the same maximum located at 224 and $222 \mathrm{~nm}$ for B3LYP and M06-2X, respectively.
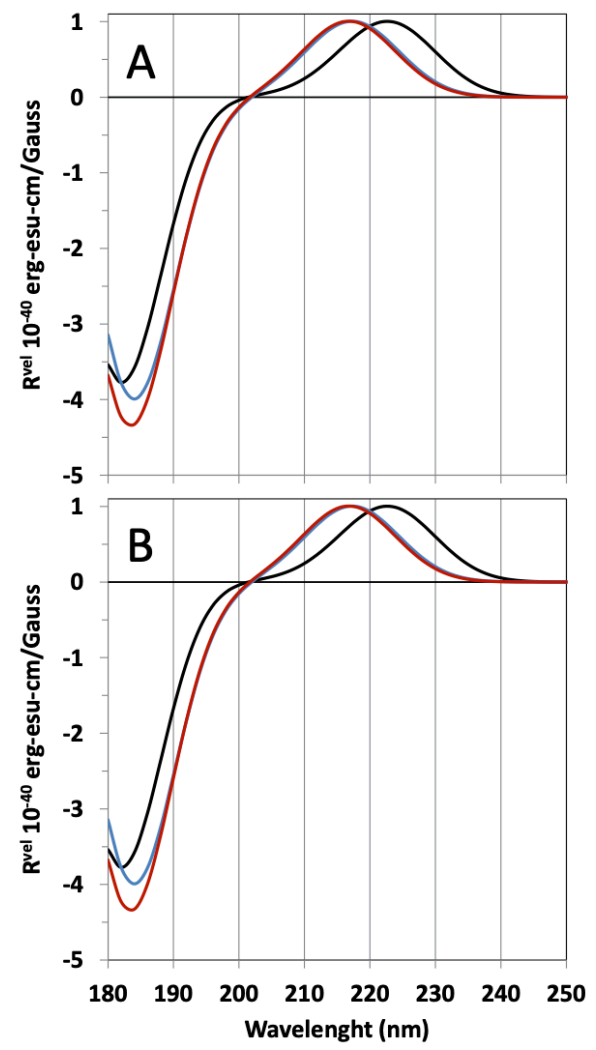

Fig. 2 Theoretical CD spectra of Ac-stbe-COOtBu monomer as a function of solvent model for acetonitrile using B3LYP (A) or M06-2X (B) theoretical level. Spectra calculated by summing $40 \%$ of the cis spectrum with $60 \%$ of the trans one. Black: gas phase (no solvent); blue: SMD solvent model; red: IEFPCM solvent model. 
On the contrary, a difference of $10 \mathrm{~nm}$ is observed for the minimum located on lower wavelength with a minimum located at 192 and $182 \mathrm{~nm}$ for B3LYP and M06-2X, respectively. When solvent effects are taken into account, no major difference is observed when employing either SMD or IEFPCM models. The maximum is blue shifted (hypsochromic effect) by 4 to $8 \mathrm{~nm}$ while the minimum remains almost unchanged (difference of 2 $\mathrm{nm}$ or less compared to gas phase spectra). If intensities are considered, a slight difference is observed between each computational method while in the gas phase, the inclusion of solvent effects reduces this effect.

Direct comparison with experimental data is difficult because Ac-stbe-COOtBu displayed only weak $C D$ signals in acetonitrile with no well-defined maxima. ${ }^{4}$ However, the simulated spectra already prefigure the shape of $C D$ spectra of longer oligomers. Experimentally, CD spectra of longer oligomers exhibit two minima near 188 and $225 \mathrm{~nm}$ and a maximum near $209 \mathrm{~nm}$. If theoretical CD spectra including acetonitrile solvent effects (IEFPCM model) are considered, cis and trans isomer exhibit specific spectral signature as shown in Figure 3.
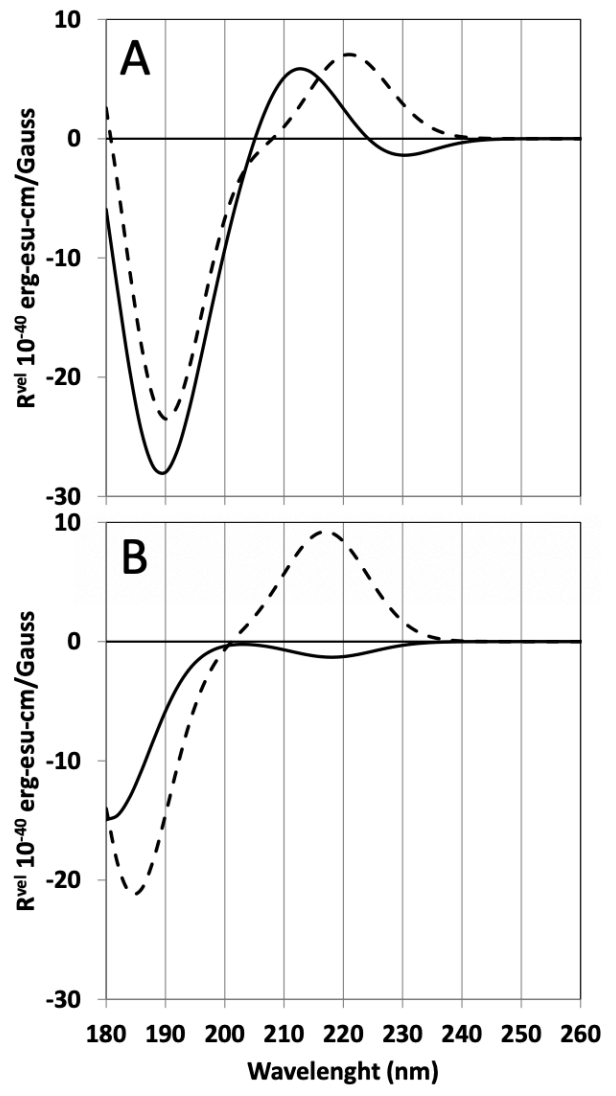

Fig. 3 Theoretical CD spectra of Ac-stbe- $\mathrm{CO}_{2} \mathrm{tBu}$ monomer using IEFPCM solvent model for acetonitrile. (A) B3LYP and (B) M06-2X. Dotted Line: trans isomer; Plain Line: cis isomer. The same scaling factor has been applied for all intensities.

On one hand, the trans isomer is not characterized by a minimum in the wavelength region around $225 \mathrm{~nm}$. On the other, a maximum is theoretically observed in this region, eliminating thus the trans conformation in longer oligomers. Regarding the cis isomer, M06-2X functional behave more badly, with a systematic blue shift around $8 \mathrm{~nm}$ (between 0.2 and $0.3 \mathrm{eV}$ ) while B3LYP exhibits a red shift around 2-3 nm (0.1 $\mathrm{eV}$ or less). This validates our choice of using B3LYP DFT functional with IEFPCM solvent model (or SMD as both behave identically). Finally, it appears that the region located around $190 \mathrm{~nm}$ (a minimum on experimental data) is a spectral signature of the conformation around the $\chi 1$ angle. Indeed, if this angle is set close to the experimental value around $-100^{\circ}$ (Figure $4 A$ ) the $C D$ signal is negative while positive if this angle is set to $+100^{\circ}$ (Figure 4B). This significantly modifies the shape of the spectrum (Figure 5). In order to understand this behaviour, NBO analysis 27,28 have been carried out on the two conformers with $\chi 1$ angle $=-100^{\circ}$ and $+100^{\circ}$. Based on natural population analysis, it appears that $\mathrm{NC}^{\alpha}$ carbon and its attached hydrogen atom are the only atoms that exhibit noticeable partial charges differences between the two structures. While this carbon atom exhibits a charge variation of $0.03 \mathrm{e}$, the charge of the hydrogen atom is modified by $0.05 \mathrm{e}$. These charge modifications induce an increase of 0.08 e of the charge separation on this specific $\mathrm{CH}$ bond when for $\chi 1=-100^{\circ}$.
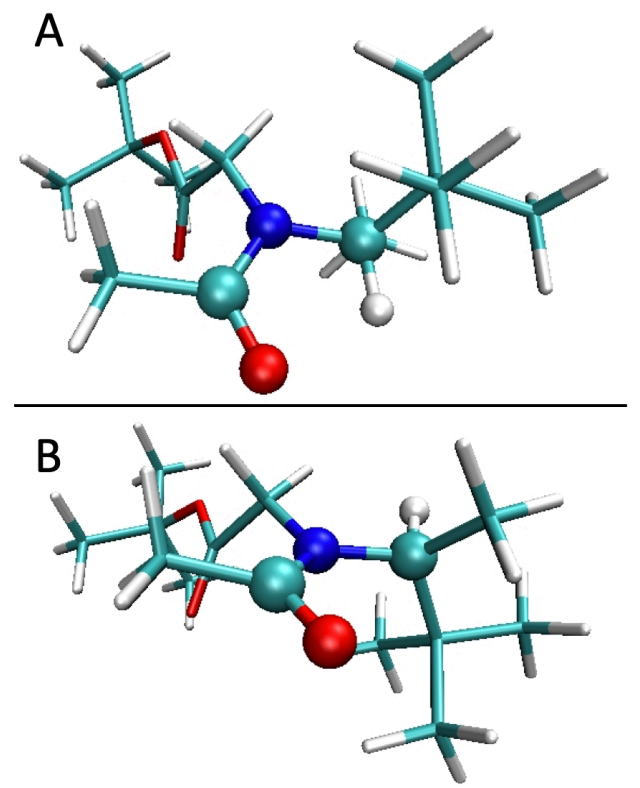

Fig. 4: 3D representation of Ac-stbe- $-\mathrm{CO}_{2} \mathrm{tBu}$. (A) $\chi 1$ angle $\sim-100^{\circ}$; (B) $\chi 1$ angle $\sim 100^{\circ}$.

More importantly, such conformation induces a dipole-dipole interaction between the carbonyl $\mathrm{CO}$ bond and the $\mathrm{CH}$ bond of the sidechain stereocenter (see Figure $4 \mathrm{~A}$ vs figure $4 \mathrm{~B}$ ). Second order perturbation analysis of the Fock matrix within the NBO approach clearly shows that there is an $n$ to $\sigma^{*}$ interaction between the lone pair orbital of the $\mathrm{CO}$ bond with the antibonding $\sigma$ orbital of the $\mathrm{CH}$ bond when $\chi 1$ angle $=-100^{\circ}$. When $\chi 1$ angle is changed, this interaction is absent.

$C D$ computations on $\mathrm{Ac}-(\text { stbe })_{n}-\mathrm{CO}_{2} t \mathrm{Bu}$ longer oligomers. Based on our preliminary study on monomer, the simulation of the $C D$ spectrum of longer Ac-(stbe) ${ }_{n}-\mathrm{CO}_{2} \mathrm{tBu}$ ( $n=2$ to 6 ) foldamers have been performed using $\mathrm{B} 3 \mathrm{LYP} / 6-31+\mathrm{G}(d, p)$ computational approach and IEFPCM to model acetonitrile as a solvent. For all systems, all-cis configurations have been considered and $\chi 1$ angle has been set close to $-100^{\circ}$. As the size of the oligomer increases, it was necessary to enlarge the number of excited 
states that was needed to be calculated. As it is presented in the ESI (Figure S1), large wavelengths region of the spectrum above $215 \mathrm{~nm}$ is not affected by the number of states calculated. On the contrary, for region below $215 \mathrm{~nm}$, the number of states has a significant impact on the shape of the spectrum. For long oligomers, if not enough states are computed, the spectrum is not in accordance with the experimental data.



Fig. 5 Theoretical CD spectra of Ac-stbe- $\mathrm{CO}_{2} \mathrm{tBu}$ monomer calculated at the B3LYP level using IEFPCM solvent model for acetonitrile. Dotted Line: $\chi 1 \approx+100^{\circ}$; Plain Line: $\chi 1 \approx$ $100^{\circ}$. The same scaling factor has been applied for all intensities.

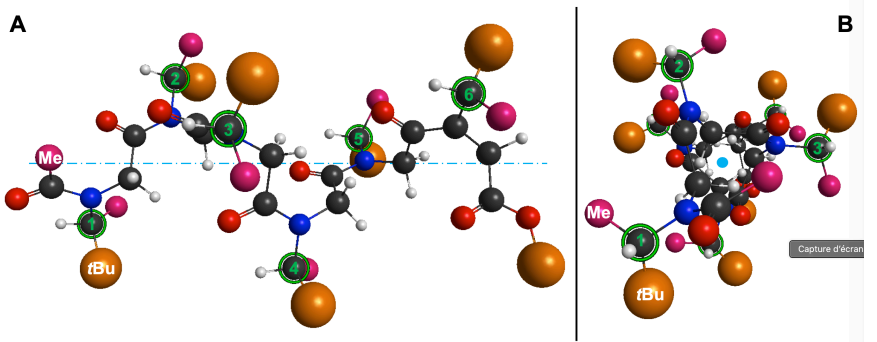

Fig. $63 \mathrm{D}$ reprenstation of $\mathrm{Ac}-(\text { stbe })_{6}-\mathrm{CO}_{2} \mathrm{tBu}$. A: side-view; B: view along helix axis depicted in blue. Chiral centers have been highlighted in green. Residue index is given in green on this atom. For the sake of clarity, tBu and Me groups are respectively represented by orange and pink spheres.

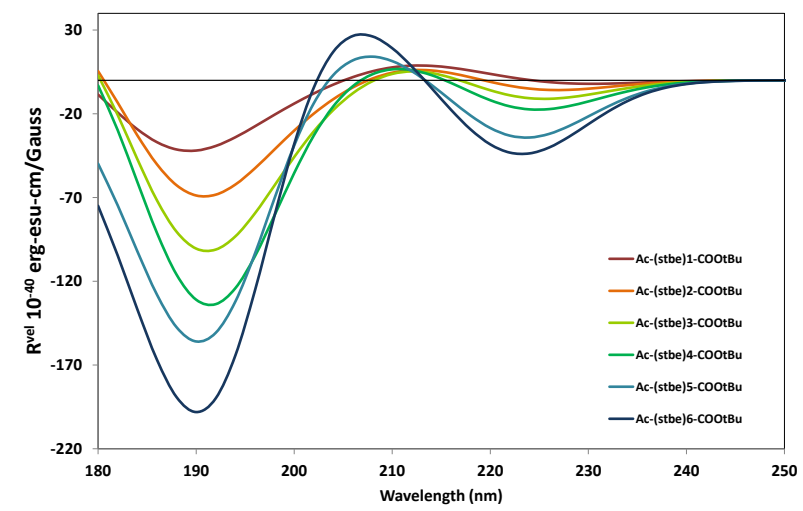

Fig. 7 Theoretical $C D$ spectra of Ac-(stbe) ${ }_{n}-\mathrm{CO}_{2} \mathrm{tBu}$ as a function of foldamers size (from $n=1$ to $n=6$ ). Only all cis conformations have been considered.
This is easily understandable by the nature of molecular orbitals involved during electronic excitation processes. In the region of interest, between 180 and $230 \mathrm{~nm}$, all electronic transitions involve $\mathrm{CO} \pi$ and $n$ type occupied orbitals and $\mathrm{CO} \pi^{*}$ unoccupied ones or combinations of $\pi^{*}$ orbitals. Hence, as the oligomer size extend, the number of $\mathrm{CO}$ carbonyl orbitals increases leading to a growing number of possible excited states that has to be considered to correctly simulate the $C D$ spectrum. While 10 to 20 states are sufficient to simulate the CD spectra of short oligomers, 100 states or more are mandatory to converge the $C D$ spectrum shape for longer oligomers. An alternative method based on complex polarization propagator has been suggested in or to circumvent this issue, ${ }^{29,30}$ however this formalism was shown to induce redshifts in some cases. ${ }^{31}$

CD spectra of all oligomers are reported in Figure 7. For longer oligomers, theoretical spectra are almost identical to experimental spectra (see Figure 4 in Roy et al.). ${ }^{4}$ The match between experimental and theoretical spectra of long oligomers allows us to confirm the 3D structure of such molecular systems. As for Ac-(stbe) ${ }_{5}-\mathrm{CO}_{2} \mathrm{tBu}$ whose 3D X-Ray structure is known, Ac(stbe) ${ }_{n}-\mathrm{CO}_{2} \mathrm{tBu}$ oligomers are all characterized by a righthanded polyproline type-I helix conformation with approximatively 3 residues per turn and a pitch theoretically estimated at $6.8 \AA$. This value is slightly larger than the experimental one determined closed to $6 \AA$. This difference can be attributed to the effect of crystal packing that is not considered in our calculations. Selected characteristic angles $\omega$, $\psi, \varphi$ and $\chi 1$ (defined as follow: $\omega=\mathrm{C}_{\alpha}(\mathrm{i}-1)-\mathrm{C}(\mathrm{i}-1)-\mathrm{N}(\mathrm{i})-\mathrm{C}_{\alpha}(\mathrm{i}), \varphi=$ $\mathrm{C}(\mathrm{i}-1)-\mathrm{N}(\mathrm{i})-\mathrm{C}_{\alpha}(\mathrm{i})-\mathrm{C}(\mathrm{i}), \psi=\mathrm{N}(\mathrm{i})-\mathrm{C}_{\alpha}(\mathrm{i})-\mathrm{C}(\mathrm{i})-\mathrm{N}(\mathrm{i}+1)$ and $\chi 1=\mathrm{C}(\mathrm{i}-1)-\mathrm{N}(\mathrm{i})-$ $\left.\mathrm{NC}_{\alpha}(\mathrm{i})-\mathrm{NC}_{\beta}(\mathrm{i})\right)$ are given in Table S1. All $\chi 1$ angle values are identical to the experimental ones with differences of less than 2 degrees. For the angles that characterize the backbone, $\omega$ and $\psi$ angles are theoretically underestimated with differences up to $5^{\circ}$ and $10^{\circ}$ for $\omega$ and $\psi$ angles, respectively, compared to the Ac-(stbe) $)_{5}-\mathrm{CO}_{2} \mathrm{tBu} X$-Ray structure. The $\varphi$ angles are overestimated by $5^{\circ}$. These cumulated angle errors can explain the difference of pitch observed for theoretical structures relative to the experimental one. Despite these differences, the geometries of all oligomers are very close.

In order to obtain further insight into the structures and the $C D$ spectral signatures of $\mathrm{Ac}$-(stbe) $\mathrm{n}_{\mathrm{n}}-\mathrm{CO}_{2} \mathrm{tBu}$ oligopeptoids, we have optimized left-handed type helices by changing the $\varphi$ angles from c.a. $-70^{\circ}$ to $+70^{\circ}$. From an energetic point of view, for a given degrees of oligomerization, a right-handed helix is more stable than left-handed one. The energy difference uniformly increases from 2.0 to $6.0 \mathrm{kcal} \mathrm{mol}^{-1}$ when going from the dimer $(n=2)$ to the hexamer $(n=6)$. Optimized angles of these oligopeptoids are given in Table S2. Interestingly, when moving to left handed helix, $\omega$ angles are roughly smaller by $10^{\circ}$ and $\psi$ angles exhibit almost $20^{\circ}$ difference relative to right-handed helix (from $-172^{\circ}$ to $+172^{\circ}$ ). Similarly, left-handed configuration impacts the $\chi 1$ angle with a modification of $10^{\circ}$. Concerning CD spectra, left-handed helix have a different spectral signature. Few differences appear in the region of 190-195 $\mathrm{nm}$ that is consistent to the conformation of the residue moiety around the $\mathrm{N}-\mathrm{C}$ bond (characterized by similar $\chi 1$ angle than the righthanded helix). On the contrary, the minimum located at $225 \mathrm{~nm}$ 
for right-handed helix has disappeared and is replaced by a maximum in the left-handed structure. This indicates that the folding and the helicity characterized in the crystal is maintained in solution.

Concerning Ac-(stbe) $)_{6}-\mathrm{CO}_{2} \mathrm{tBu}$, the calculation of $\mathrm{CD}$ spectrum as a function of $\chi 1$ angles has been performed by switching progressively these angles from c.a. $-100^{\circ}$ to $90^{\circ}$.

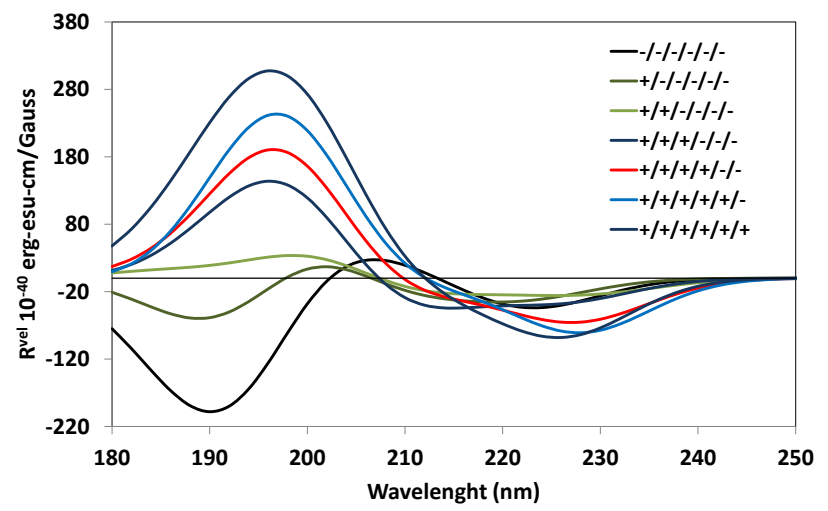

Fig. 8 Theoretical CD spectra of Ac-(stbe)6-CO2tBu as a function of different $\chi 1$ angles. A (-) sign indicates a $\chi 1$ angle around $-100^{\circ}$ and a (+) sign indicates a $\chi 1$ angle around $90^{\circ}$.

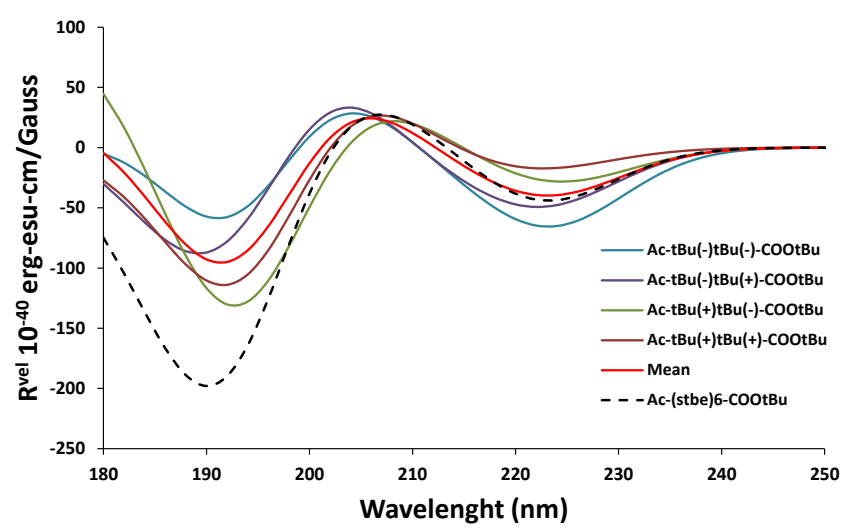

Fig. 9 Theoretical CD spectra of $\mathrm{Ac}-(\text { stbe })_{6}-\mathrm{CO}_{2} \mathrm{tBu}$ (dotted) and Ac-tBu-(stbe) $)_{4}$-tBu$\mathrm{CO}_{2} \mathrm{tBu}$ (plain) as a function of $\varphi$ angles values of tBu moieties.

The $C D$ spectra are given in Figures $1 \mathrm{~B}$ and 8 depending on the number of negative or positive $\chi 1$ angles. As expected, while the number of residues with positive $\chi 1$ value increases, the shape of the spectra is modified. More precisely, the region around $190 \mathrm{~nm}$ is progressively modified when going from all negatives $\left(-100^{\circ}\right)$ to all positives $\left(+90^{\circ}\right) \chi 1$ values. As already observed for the monomer, the signal in this region progressively diminish and from a negative signal ended into a positive one.

$C D$ computations on $A c-(t B u)_{m}-(s t b e)_{4}-(t B u)_{m}-C_{2} t B u$ heterooligomers. Finally, the last step in the exploration of $\mathrm{CD}$ spectra of $\mathrm{Ac}$-(stbe) $)_{n}-\mathrm{CO}_{2} \mathrm{tBu}$ foldamers is the investigation of the hetero-oligomer $\mathrm{Ac}-(\mathrm{tBu})_{\mathrm{m}}-(\mathrm{stbe})_{4}-(\mathrm{tBu})_{\mathrm{m}}-\mathrm{CO}_{2} \mathrm{tBu}$ synthesized by the group of Taillefumier. ${ }^{4}$ Among the foldamers experimentally reported, we have considered Ac-(tBu)-(stbe) $4^{-}$ $(\mathrm{tBu})-\mathrm{CO}_{2} \mathrm{tBu}$ that enables a direct comparison with $\mathrm{Ac}-(\mathrm{stbe})_{6}-$ $\mathrm{CO}_{2} \mathrm{tBu}$ previously calculated.
Four geometries with different combination of $\varphi$ angles for the tBu residues have been optimized. These angles have been set either positive or negative, the ones of stbe moieties remaining to their optimal negative values. As a notation, these structures are denoted: $\mathrm{Ac}-\mathrm{tBu}(-) \mathrm{tBu}(-)-\mathrm{CO}_{2} \mathrm{tBu}, \mathrm{Ac}-\mathrm{tBu}(-) \mathrm{tBu}(+)-\mathrm{CO}_{2} \mathrm{tBu}$, $\mathrm{Ac}-\mathrm{tBu}(+) \mathrm{tBu}(-)-\mathrm{CO}_{2} \mathrm{tBu}, \mathrm{Ac}-\mathrm{tBu}(+) \mathrm{tBu}(+)-\mathrm{CO}_{2} \mathrm{tBu}$. The energy of these four optimized isomers are within $0.4 \mathrm{kcal} \mathrm{mol}^{-1}$ from each other. In terms of geometry, $\varphi$ angles for tBu residues are larger than for stbe ones $\left(86^{\circ}\right.$ vs $\left.72^{\circ}\right)$. A negative value for the tBu residue located at the acetyl extremity is energetically slightly more favorable. These geometrical features are consistent with those previously obtained an all tBu foldamers ${ }^{32}$ and highlight the flexibility of the two extremity of this oligomers.

Circular dichroism spectra calculated for these four structures are depicted in Figure 9. A significant decrease of intensity is observed for the minima near $190 \mathrm{~nm}$ compared to Ac-(stbe) $6_{6}$ $\mathrm{CO}_{2} \mathrm{tBu}$. This decrease is more important if negative torsion value is set for the acetyl extremity. This is associated to a significant increase of the signal observed for the minima near $224 \mathrm{~nm}$. On the contrary, for a positive torsion value at the acetyl extremity, the decrease at $190 \mathrm{~nm}$ is less pronounced and is associated to a decrease of the signal at $224 \mathrm{~nm}$. This is qualitatively in accordance with the experimental trend. 4 Regarding the energy difference between all four optimized structures, the average CD spectrum has also been calculated. A significant decrease in intensity of the minima near $190 \mathrm{~nm}$ is observed relative to $\mathrm{Ac}$-(stbe) $)_{6}-\mathrm{CO}_{2} \mathrm{tBu}$ while the rest of the curves remained broadly unchanged if we allow ourselves to paraphrase the authors of the experimental study.

Molecular dynamics simulations of $A c-(t B u)_{m^{-}}(s t b e)_{4}-(t B u)_{m^{-}}$ $\mathrm{CO}_{2} t \mathrm{tBu}$ hetero-oligomers. In order to go beyond the interpretation of $C D$ spectra computed for combinations of local conformations, 50 ns classical molecular dynamics simulations were performed for $\mathrm{Ac}-(\mathrm{tBu})_{3}-(\mathrm{stbe})_{4}-(\mathrm{tBu})_{3}$-COOtBu decamer. For this system, the main objective of this part is to compare the dynamical behavior of chiral vs achiral residues and ultimately, if possible, the ability of the (stbe) $)_{4}$ chiral sequence to induce chirality in the helicoidal folding of the $(\mathrm{tBu})_{3}$ tags. In that perspective, four different conformations of the peptoid were simulated. The conformations were generated based upon the $\varphi$ dihedral angle. ${ }^{32}$ While keeping the $\varphi$ angle value of stbe monomers around $-90^{\circ}$, we have generated the conformations by changing the $\varphi$ angle sign of tBu monomers to $+90^{\circ}$ or $-90^{\circ}$. The four conformations generated have the following $\varphi$ angle signatures $(-)_{10},(+)_{3}-(-)_{4}(+)_{3},(+-+)-(-)_{4}-(+-+)$ and $(-+-)-(-)_{4}-(-$ $+-)$.

Firstly, a comparison of the mean structures obtained by molecular dynamic of the hetero-oligomers with the structures previously obtained either by quantum chemistry methods or by X-Ray diffraction of $\mathrm{Ac}-(\mathrm{tBu})_{2}-(\mathrm{stbe})_{4}-(\mathrm{tBu})_{2}-\mathrm{CO}_{2} t \mathrm{Bu} u^{4}$ was carried out on the basis of the part of the structure which contains only stbe fragments. If the representative dihedrals angles are considered, the mean molecular dynamic structures are quantitatively coherent with previous results with $\omega, \psi, \varphi$ and $\chi 1$ equal to $-8.1^{\circ} \pm 10.1^{\circ},-72.6^{\circ} \pm 8.6^{\circ}, 178.1^{\circ} \pm 12.1^{\circ}$ and $97.9^{\circ} \pm 7.6^{\circ}$, respectively (See Table S3 for more details on $(-)_{10}$ and X-Ray structure). 
Figure S2 to S5 show the probability distribution of dihedral angles during the $50 \mathrm{~ns}$ classical simulation. The $\omega$ angle values for peptoid bonds between two stbe monomers are found to have very similar values from their distribution curves for all four conformations. In contrast, the $\omega$ angle between tBu monomer shows different distributions for different conformations. Torsion distribution peaks have either $+10^{\circ}$ or $10^{\circ}$ value depending upon the sign of the $\varphi$ angle for the concerned tBu residue. This result is in accordance with our previous observation on the folding of homo oligopeptoids supporting a tBu side chain. ${ }^{32}$ Moreover, distribution of $\omega$ angle between $\mathrm{tBu}$ and stbe monomers are also dependent on the $\varphi$ angle sign of the tBu. A distinct difference can be seen in the $\varphi$ angle distribution between tBu and stbe monomers. In agreement with the folding of homo oligopeptoids supporting a tBu side chain, ${ }^{32}$ the peak of the $\varphi$ angle distribution is around $90^{\circ}$ or $-90^{\circ}$ depending on the residue conformation. As already mentioned, ${ }^{32}$ the sign of the angle does not change during classical simulations. In contrast, for stbe residues, the peak in the $\varphi$ angle distributions is around $-75^{\circ}$ and remains negative during simulations. Similarly, distribution peak of the $\psi$ of tBu monomers is observed around $\pm 170^{\circ}$. In some cases, this peak shifts around $\pm 160^{\circ}$ with a high probability of occurrence near $\pm 170^{\circ}$. For stbe residues the $\psi$ angle distribution follows similar trend to that of tBu monomers.

Replica Exchange Molecular Dynamics (REMD) simulations were performed on the oligomer using all four $(-)_{10},(+)_{3}-(-)_{4}(+)_{3},(+-$ $+)-(-)_{4}-(+-+)$ and $(-+-)-(-)_{4}-(-+-)$ conformations. These simulations were performed at temperature range of $300-600 \mathrm{~K}$ with each replica simulated for 50 ns. ${ }^{32}$ The results for the dihedral angle distribution during REMD are shown in Figures S6 to S9. From $\omega$ angles distribution, it can be observed that some of the peptoid bonds do venture into trans conformations during the REMD simulations. However, this phenomenon is negligible and can only be observed for peptide bonds towards C-terminus of the oligopeptoids. Switch of $\varphi$ angle values from positive to negative and vice versa can be observed during REMD simulations. This switch is more pronounced for the tBu monomers in comparison to stbe moiety for which negligible shift is observed. However, the presence of chiral residues in the middle of the oligopeptoid sequence might be the reason for the decrease in $\varphi$ angle switch occurrence. The comparison of the $\varphi$ angle distribution during REMD simulations of the decapeptoids under study with that of the homo octamer supporting a tBu side chain ${ }^{32}$ is shown in Figure S10. It confirms that the switch in the sign of $\varphi$ angle values during the REMD simulations is position dependent. The monomers towards the termini are more susceptible to this sign switching in comparison to inner monomers. However, though extended, the sampling is not large enough to clearly demonstrate that the chiral sequence induces the helicity to the achiral tags. Regarding the $\psi$ angle distribution, no significant change was observed during the REMD simulations. However, homogenization of this distribution occurred. In other words, the peak in distribution found around $\pm 160^{\circ}$ for some tBu vanishes and maximum probability of distributions for all tBu are found around $\pm 170^{\circ}$.
Finally, we have tried to highlight the key intramolecular interactions from which the asymmetric folding originates from. We have previously shown the significance of weak interactions in determining the folding of oligopeptoids with different side chains into well-definite secondary structures. ${ }^{32}$ Similar weak interactions between various groups during the MD simulations were quantified for mixed tBu-stbe decamer. Figures S11 to S14 show probability plot of such interactions during the simulations. Figure S11 shows the probability of backbone carbonyl oxygen forming interaction with methylene backbone i.e. $\mathrm{CH} \cdots \mathrm{O}=\mathrm{C}$ interactions. Interactions are observed between carbonyl oxygen with methylene group of monomers one/two residue before ( $\mathrm{i} \rightarrow \mathrm{i}-1 / \mathrm{i}-2$ ). Interestingly $\mathrm{i}$ to $\mathrm{i}-2$ interactions are more pronounced when the carbonyl group of a stbe residue is involved in the interaction. Additionally, the probability of this interactions is found higher in comparison to their tBu counterparts.

Figure $\mathrm{S} 12$ depicts the probability of interactions between sidechains, $\mathrm{CH}_{3}$ as well as $\mathrm{CH}$ for stbe, of different residues during the simulations. For stbe side chain, the probability was averaged over all three $\mathrm{CH}_{3}$ and one $\mathrm{CH}$ groups. It clearly appears that the sidechains of stbe residues form more interaction with other sidechains in comparison to tBu. Also the stbe side chain have similar probability for all four type of decamer conformations, with very low interaction probabilities for $6^{\text {th }}$ and $7^{\text {th }}$ residues in $(+-+)(-)_{4}(+-+)$ and $(-+-)(-)_{4}(-+-)$ conformations respectively. Distinct difference can be seen for interaction probabilities of $\mathrm{tBu}$ side chain for different conformations. This agrees with our previous results on pure tBu octamer where similar $\varphi$ angle conformation, $(-)_{8}$, had more such interactions in comparison to alternate $\varphi$ angle conformation, $(+-)_{4} \cdot{ }^{32}$

In Figures S13 and S14, we represent probabilities of interaction between main chain carbonyl oxygen with side chain $\mathrm{CH}_{3}$ group one residue ahead. For stbe, three methyl groups attached to the same carbon are considered and it gives similar vibes to that of tBu. The fourth methyl group in the sidechain was not considered in these plots. Negligible interaction of this separate methyl group of stbe with any of the carbonyl oxygen was observed (results not shown). Coming back to Figures S13 to S16, minimal difference is observed in the interaction probabilities of main chain carbonyl oxygen with sidechain methyl groups for different conformations. This is in accordance with our previous results on the folding of octamer supporting only a tBu side chain. ${ }^{32}$ Moreover, in all four conformations the carbonyl oxygen of the inner residues establishes a smaller amount of weak interactions.

Another important interaction involves backbone carbonyl of residues (i) and ( $i-1)$. For all four conformations this interaction was out to be omnipresent. This is in accordance with our previous results where such interactions are omnipresent when two $\alpha$-peptoid monomers are concerned. ${ }^{33}$ Some amount of backbone (i) $\mathrm{C}=\mathrm{O} \cdots(\mathrm{i}+1) \mathrm{C}=\mathrm{O}$ interactions are also observed during the simulations. The last type interactions studied in the present work is that between main chain carbonyl oxygen and side-chain chiral carbon. It is observed that for all four conformations during one third of the simulation time the main 
chain carbonyl oxygen forms interaction with side-chain chiral carbon of the monomer placed one residue ahead in the chain.

\section{Conclusions}

In conclusion, we demonstrate that the simulation of electronic CD spectra can be finely achieved by TD-DFT calculations without resort to any empirical corrections providing that enough states are computed and that an appropriate implicit solvent model is used. The systematic simulation of $C D$ spectra of a set of conformers not only allow to ascribe the structural features that contributes to the $C D$ signal but, in fine, enables to determine conformational features of the molecule under study at the atomic scales. This modelling strategy has been fruitfully applied to investigate the folding of chiral $\alpha$ oligopeptoids.

This spectroscopic investigation has been completed by classical MD simulations whose analysis reveals that the helical structure results from attractive weak interactions between stbe side chains, between backbone methylene and carbonyl groups, and between two backbone carbonyl groups. Interestingly, due to its appropriate bulk, more London interactions can be developed between tertiobutylethyl side chains than between tertiobutyl side chain. This suggested, that the conformation and the helicity of the chiral sequence is locked, whereas the achiral tags are more flexible and can adapt their conformation to the one induced by the chiral sequence.

\section{Conflicts of interest}

There are no conflicts to declare.

\section{Acknowledgements}

This work was supported by a grant overseen by the French National Research Agency project ARCHIPEP. L.P. and N.B. thank CCIR of ICBMS and P2CHPD of Univ. Lyon 1 for providing computational resources. N.B. and F.J. thank the CALcul en MIdi-Pyrénées (CALMIP, grant P0758) for generous allocations of computer time.

\section{Notes and references}

1 K. Kirshenbaum, A. E. Barron, R. A. Goldsmith, P. Armand, E. K. Bradley, K. T. V. Truong, K. A. Dill, F. E. Cohen and R. N. Zuckermann, Proc. Natl. Acad. Sci. U. S. A., 1998, 95, 43034308.

2 K. Kirshenbaum, Structures - Web page, https://wp.nyu.edu/kirshenbaumlab/peptoiddbstructures/.

3 S. Khrapunov, Anal. Biochem., 2009, 389, 174-176.

$4 \quad$ O. Roy, G. Dumonteil, S. Faure, L. Jouffret, A. Kriznik and C. Taillefumier, J. Am. Chem. Soc., 2017, 139, 13533-13540. J. R. Stringer, J. A. Crapster, I. A. Guzei and H. E. Blackwell, J. Am. Chem. Soc., 2011, 133, 15559-15567.
Commun., 2015, 6, 1-10. 369-375. CT, 2016, 2016. 789. Phys., 1980, 72, 650-654. 5639-5648. 215-241. Res., 2011, 39, 511-517. 191. 96, 1-4. 151. $B, 2009,113,6378-6396$. 3210-3221. 4073. 1985, 83, 735-746. 20535. 134102.
B. C. Gorske, J. R. Stringer, B. L. Bastian, S. A. Fowler and H. E. Blackwell, J. Am. Chem. Soc., 2009, 131, 16555-16567.

P. Armand, K. Kirshenbaum, A. Falicov, R. L. Dunbrack, K. A. Dill, R. N. Zuckermann and F. E. Cohen, Fold. Des., 1997, 2,

H. Aliouat, C. Caumes, O. Roy, M. Zouikri, C. Taillefumier and S. Faure, J. Org. Chem., 2017, 82, 2386-2398.

C. Caumes, O. Roy, S. Faure and C. Taillefumier, J. Am. Chem. Soc., 2012, 134, 9553-9556.

M. J. Frisch, G. W. Trucks, H. B. Schlegel, G. E. Scuseria, M. A. Robb, J. R. Cheeseman, G. Scalmani, V. Barone, G. A. Petersson, H. Nakatsuji, X. Li, M. Caricato, A. Marenich, J. Bloino, B. G. Janesko, R. Gomperts, B. Mennucci, H. P. Hratchian, J. V. Ort and D. J. Fox, Gaussian, Inc., Wallingford

A. D. Becke, J. Chem. Phys., 1993, 98, 5648-5652.

C. Lee, W. Yang and R. G. Parr, Phys. Rev. B, 1988, 37, 785-

R. Krishnan, J. S. Binkley, R. Seeger and J. A. Pople, J. Chem.

A. D. McLean and G. S. Chandler, J. Chem. Phys., 1980, 72 ,

Y. Zhao and D. G. Truhlar, Theor. Chem. Acc., 2008, 120,

J. M. Wang, R. M. Wolf, J. W. Caldwell, P. a Kollman and D. a Case, J. Comput. Chem., 2004, 25, 1157-1174.

E. Vanquelef, S. Simon, G. Marquant, E. Garcia, G. Klimerak, J. C. Delepine, P. Cieplak and F. Y. Dupradeau, Nucleic Acids

J. Wang, W. Wang, P. A. Kollman and D. A. Case, J. Mol. Graph. Model., 2006, 25, 247-260.

J. C. Phillips, R. Braun, W. Wang, J. Gumbart, E. Tajkhorshid, E. Villa, C. Chipot, R. D. Skeel, L. Kalé and K. Schulten, J. Comput. Chem., 2005, 26, 1781-1802.

H. Lei and Y. Duan, Curr. Opin. Struct. Biol., 2007, 17, 187-

D. Van Der Spoel and M. M. Seibert, Phys. Rev. Lett., 2006,

Y. Sugita and Y. Okamoto, Chem. Phys. Lett., 1999, 314, 141-

A. Patriksson and D. Van Der Spoel, Phys. Chem. Chem. Phys., 2008, 10, 2073-2077.

A. V Marenich, C. J. Cramer and D. G. Truhlar, J. Phys. Chem.

V. Barone, M. Cossi and J. Tomasi, J. Chem. Phys., 1997, 107,

A. E. Reed and F. Weinhold, J. Chem. Phys., 1983, 78, 4066-

A. E. Reed, R. B. Weinstock and F. Weinhold, J. Chem. Phys.,

P. Norman, Phys. Chem. Chem. Phys., 2011, 13, 20519-

P. Norman, M. Linares, Chirality, 2014, 26, 483-489.

A. Jiemchooroj, P. Norman, J. Chem. Phys., 2007, 126,

G. Angelici, N. Bhattacharjee, O. Roy, S. Faure, C. Didierjean, 
Journal Name

L. Jouffret, F. Jolibois, L. Perrin and C. Taillefumier, Chem. Commun., 2016, 52, 4573-4576.

33

G. Dumonteil, N. Bhattacharjee, G. Angelici, O. Roy, S. Faure,

L. Jouffret, F. Jolibois, L. Perrin and C. Taillefumier, J. Org.

Chem., 2018, 83, 6382-6396. 
Table of contents

TD-DFT quantitative simulation of

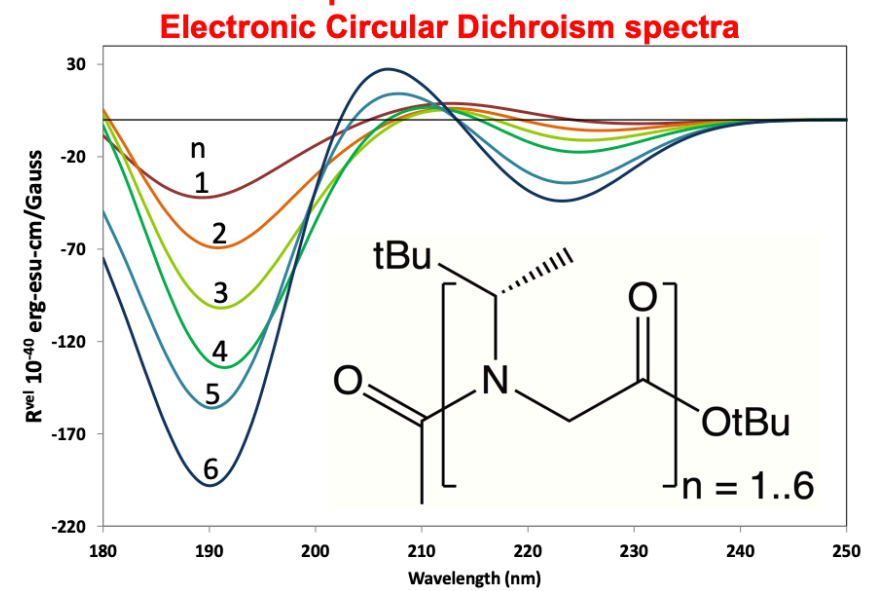

Accurate TD-DFT calculations of electronic circular dichroism have been performed to characterise the 3D structure of $\alpha$-peptoids. 